\title{
EVALUACIÓN DE LA VARIABILIDAD MORFOLÓGICA DE PROSOPIS CHILENSIS EN LA REGIÓN DE TACNA Y SU RELACIÓN CON LOS FACTORES ECOLOGICOS DEL MEDIO DESÉRTICO
}

Elsa Borujerdi'; Rosario Zegarra

\author{
RES U M EN
}

Los valles costeros de área desertica en Tacna (Perú) han sufrido un importante fenómeno de deforestación debido a la explotación agrícola, ya que estos valles constituyen una fuente de suelos fértiles de la zona costera central del gran desierto de Atacama.

El fenómeno de deforestación incluye la desaparición de muchas especies vegetales en el sur del Perú. Una de estas especies es Prosopis chilensis, árbol nativo de los ecosistemas áridos y semiáridos de América del Sur.

Sin embargo, este árbol es particularmente útil para los pobladores locales y es usado como combustible (leña y carbón), madera para construcción y sus frutos como forraje para el ganado vacuno. Por esta razón se justifica el establecimiento de un programa de conservación y reforestación de esta especie.

El principal objetivo de la presente investigación ha sido analizar la variabilidad morfológica de Prosopis chilensis así como su adaptación del medio ambiente desertico con la finalidad de mejorar su utilización en los programas de reforestación. Sin embargo, la investigación realizada con bajo número de individuos actualmente creciendo en el departamento de Tacna no hizo posible establecer una significante correlación entre la variabilidad morfológica y las condiciones ecológicas. A pesar de esto, los árboles de Prosopis chilensis muestran una irrevocable adaptación a los particulares condiciones extremas de aridez o estres hídrico; estos atributos genéticos pueden ser explotados en un vasto programa de reforestación en las zonas áridas del sur del Perú.

Palabras Claves : variabilidad morfológica, Prosopis chilensis, zonas áridas, factores ecológicos, adaptación.

\section{$A B S T R A C T$}

The coastal valleys of the Tacna desert area (Peru) underwent an important phenomenon of deforestation subject to an agricultural aim, because these valleys constituted the unique fertile grounds of the coastal zone of Tacna covered by the desert of Atacama.

This phenomenon of deforestation involves the disappearance of many vegetable species in the area, one of these is the Prosopis chilensis, native tree of and anot semiarid ecosystem from South America. However, this tree is particularly useful for the local populations, as structural timber, and its fruits as fodder for the cattle. So it seems important to set up a program of conservation and replanting using this species.

The main objetive of the present investigation has been analyse the morphological variability of Prosopis chilensis as well as its adaptation to the desert environment in order to improve its usage in reforestation programs. However, the experimentation, carried out on a too low number of individuals actually growing in Tacna did not make it possible to establish a significant correlation between morphological variability and ecological conditions. Nevertheless, the tree of Prosopis chilensis show a remarkable adaptability to particularly extreme arid conditions, capacity which will be interesting study included in reforestation projects in arid areas of Southern Perí. adaptability.

Key words: morphological variability, Prosopis chilensis, arid zones, ecological factors,

(1) Doctora,

(2) Doctora en Botánica Agricola. docente de la Universidad Nacinal "Jorge Basadre Grohmann" Tacna - Perú. 


\section{INTRODUCCIÓN}

La zona costera de la región de Tacna, situada en el desierto de Atacama, al Sur del Perú, sufrió un importante fenómeno de deforestación en los distintos valles que la surcan, dado que estas tierras fértiles se utilizan para la agricultura. La desaparición de los bosques secos, vegetación de estos valles, implica consecuencias dañinas al nivel económico, y también ecológico.

Por una parte, los árboles de estos bosques constituyen una fuente de carbón, de madera de construcción, y también de forraje para las poblaciones locales (Morizaki Taura,A., 1998). Por otra parte, algunas especies vegetales de estos bosquecillos se ven amenazadas de extinción.

Parece pues importante proteger estos ecosistemas frágiles estableciendo proyectos de conservación y reforestación. Proyectos de este tipo ya existen en América Latina, como el « Programa Algarrobo » (del nombre común de la especie Prosopis pallida en español) establecido desde 1990 por el instituto Argentino de Investigación de las Zonas Áridas (IADIZA) y cuyo objetivo general consiste en repoblar las zonas deterioradas de clima árido templado con especies nativas del género Prosopis (Cony, M., 1998); del mismo modo, el Instituto Nacional de los Recursos Naturales (INRENA) ha establecido el "Proyecto Algarrobo" sobre la costa norte del Perú.

Prosopis chilensis (Mol.) Stuntz es una especie arbustiva nativa de Sudamérica desarrollándose en las zonas áridas y semiáridas de Chile, Perú y Bolivia (un tablero botánico que presenta la especie está disponible en el anexo). Algunos árboles de esta especie están aún presentes en la región de Tacna, a pesar de una reducción importante de individuos vinculada a la deforestación. Dada su importancia en la economía local como árbol de usos múltiples, la protección de esta especie aparece como importante. Sin embargo, un trabajo de conservación de la especie solo es posible una vez que sea claramente caracterizada a nivel morfológico y genético. Este trabajo de determinación fue objeto de numerosas investigaciones anteriores, en particular con el botánico argentino A. Burkart que publicó una monografia sobre la clase Prosopis en 1976; otros trabajos más recientes han sido efectuados también por el Instituto Argentino de Investigacion de las Zonas Aridas (IADIZA), y el Centro de Investigacion de las Zonas Áridas (CIZA Universidad Agraria La Molina, LimaPerú). Pero la variabilidad morfológica encontrada en las especies del genero Prosopis permanece aún mal definida.

El objetivo de este estudio será, pues inicialmente, caracterizar la variabilidad morfologica del Prosopis chilensis en la región de Tacna; luego, a partir del estudio de las condiciones ecológicas consustanciales a cada árbol, intentar explicar esta biodiversidad intraespecifica por una adaptación del árbol al medio.

\section{MATERIAL Y MÉTODOS}

\section{Localización de los lugares de estudio}

Inicialmente, la selección de los individuos se efectuó en los tres valles principales de la zona costera de la región de Tacna (valles de Caplina, Sama y Cinto), con el fin de trabajar en lugares que presentan las condiciones climáticas las más variadas (ver Cuadro 1). Después de una visita de reconocimiento en cada valle, los árboles se eligieron según sus características morfológicas y fenologicas (presencia/ausencia de flores $y / 0$ de frutos) que permitlan obtener un muestfeo más representativo posible de la biodiversidad encontrada.

Su distribución es la siguiente:

Valle de Caplina: distrito de Calana, 3 individuos

Distrito de Pachia, 2 individuos

Valle de Sama: 2 individuos

Valle de Cinto: 2 individuos

Un plan en anexo permite localizar geográficamente a cada individuo.

En el valle de Caplina se encuentra la ciudad de Tacna, capital de la región, es pues el valle más urbanizado. Los 3 primeros individuos situados en Calana se encuentran dentro del distrito, a $850 \mathrm{msnm}$, sobre un terreno no explotado, (ver Foto 1 en anexo). Mientras que los 2 árboles estudiados en Pachía se sitúan al exterior del distrito, aproximadamente a 1200 msnm, cerca de la carretera Tacna-Calientes, en los alrededores de una pequeña explotación agraria. Los 2 individuos de Sama no se sitúan dentro del valle, sino en altura sobre al lado norte del cerro que domina el valle, respectivamente a $650 \mathrm{msnm}$ (ind .6) y 680 msnm (ind.7) (ver Foto 2 en anexo). En el valle de Cinto, los 2 Prosopis se sitúan en la avenida aluvial del rio desecado, a distintos niveles del valle, respectivamente a $900 \mathrm{msnm}$ (ind. 8) y $650 \mathrm{msnm}$ (ind.9) (ver Fotos 3 y 4 en anexo).

\section{Condiciones climáticas}

Los datos climáticos utilizados proceden de las estaciones meteorológicas del SENAHMI (Servicio Nacional de Meteorologia e Hidrologia) de Calana y Pachía para el valle de Caplina, Sama-Inclán para el valle de Sama y Locumba para el valle de Cinto. Con el fin de obtener resultados comparables entre los valles, las medias se efectuaron a partir de los datos más recientes tomados (variable según los lugares) y 
sobre un período de 10 años. Los datos climáticos para cada lugar de estudio se presentan en el cuadro siguiente, los años escritos entre paréntesis bajo las medias corresponden al perlodo de lectura.

\section{Cuadro №1}

Condiciones climáticas de los valles de Caplina, Sama y Locumba

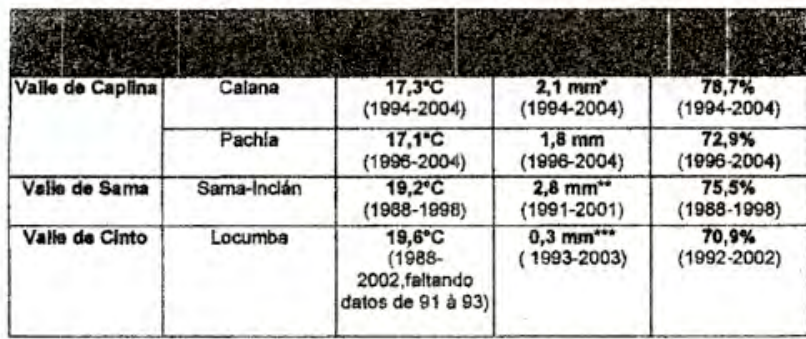

Fuentes: SENAHMI

* Las precipitaciones son escasas, la mayor parte del tiempo en forma de garua.

** Este valor fue aumentado por precipitaciones excepcionales en 1997 (hasta $48 \mathrm{~mm}$. registrado en enero), vinculado con el fenómeno del Niño (Morris Ayca, M., Panty Neyra, O., 1999).

*** Las precipitaciones son muy escasas y se registró una sequía total ( $0 \mathrm{~mm}$. de precipitación) de 1990 a 1995.

Para concluir, se puede observar que los valles de Sama y Cinto presentan temperaturas más elevadas, esto puede ser explicado por una estrecha geomorfología de los valles y la composición de sus suelos que determinan una buena recepción a los rayos solares. Además, en el valle de Cinto el cielo está despejado la mayor parte del año. Por otra parte, el valle de Caplina y el de Sama tienen un clima claramente más húmedo que el valle de Cinto. Además, la sequila de este valle se vio aumentada, desde hace 40 años, por el drenaje del río a causa de la explotación de la laguna de Suche, su alimentación hídrica, por la industria minera.

Observación: El fenómeno del Niño, cuyas últimas frecuencias tuvieron lugar en 1989-90, 1991-92 y 1997 98 , implica perturbaciones climáticas que se traducen en la región costera por elevadas precipitaciones y temperaturas extremas, condiciones que permiten la regeneración de los bosques secos, (Morizaki Taura, A. , 1998).

\section{Metodos:} Selección y medida de las variables
morfológicas

Las variables estudiadas se eligieron basándose en trabajos de investigación anteriores sobre el análisis morfológico de árboles del género Prosopis (Aguilera, A.; Bobadilla, D.; Cortés, R.; Vargas, H., 1986). A continuación, se efectuó un trabajo de selección con el fin de no conservar más que las variables que presentaban diferencias notables entre los individuos.

\section{Las variables conservadas son las siguientes:}

Variables relativas a la arquitectura del árbol:

Altura total (A. Tot)

Altura del tronco (A. Tronc)

Área de copa (Área. Copa)

\section{Variables relativas a las hojas:}

Longitud media de las pinnas (L.Med.Pin)

Número de pares de folílos por pinnas (Num.Par.Fol)

Longitud media de los follolos (Long. Fol)

Ancho medio de los foliolos (Anch. Fol)

Espacio entre follolos (Esp. Fol)

Cantidad de ramas jóvenes (Ram. Jóv), cuantificado con una escala creciente de 1 a 3 . (fase 1 = abscisión casi total de las hojas u hojas amarillas, muy pocas ramas jóvenes; fase 2 = ramas del año anterior mayoritarios; fase 3 = nuevas ramas en cantidad bastante importante

Variable relativa a la floración (flores):

Presencia (P)/Ausencia $(A)$ de flores

Variable relativa a la fructificacion (frutos):

Presencia $(P) / a u s e n c i a(A)$ de frutos

Para cada individuo, las medidas relativas a la arquitectura del árbol se efectuaban in situ, lo mismo ocurre con las observaciones relativas a la floración y la fructificación.

Las medidas sobre las hojas se efectuaban a partir de muestras de ramas recogidas de manera homogénea sobre el árbol (al Norte, al Sur, al Este y el Oeste; en parte superior e inferior). Para el cálculo de L.Med.Pin, Long. Fol, Anch. Fol y Esp. Fol las medias se efectuaban sobre 5 hojas que representaban lo mejor 
posible la biodiversidad morfológica de las hojas en el árbol. El material recogido estaba secado para constituir un herbario que ilustraba la variabilidad encontrada en las ramificaciones, hojas, flores y frutos.

\section{Estudio de los factores edáficos}

La selección de los caracteres de suelo a estudiar se efectuó considerando las variables supuestas como las más influyentes sobre el crecimiento del árbol, es decir, el PH, la conductividad eléctrica (Cond. Eléc., medida en milimhos $/ \mathrm{cm}$ a $25^{\circ} \mathrm{C}$ ), la textura y el porcentaje de grava (Porc.Grav, particulas $>2 \mathrm{~mm}$ ).

Con el fin de tomar las muestras, un corte de $30 \mathrm{~cm}$ de profundidad se efectuaba al pie del árbol, y se tomaba la muestra de manera homogénea a lo largo del corte, en razón de $1 \mathrm{~kg}$ de suelo por lugar.

Luego, las medidas de $\mathrm{pH}$ y conductimetría se adoptaban en el laboratorio de suelo de la Universidad de Tacna, a partir de soluciones de suelo (las proporciones utilizadas son respectivamente 1:2.5 para el análisis de $\mathrm{pH}$ y 1:1 para el análisis de conductimetría).

Para el análisis de textura la solución de suelo se preparaba con tierra tamizada al tamiz $N^{\circ} 10$ (partículas $>2 \mathrm{~mm}$ ) y con hexametafosfato de sodio, $y$ luego se mezclaba con ayuda de un agitador mecánico. Las medidas relativas al porcentaje de arena, limo y arcilla se median con un hidrómetro. La textura se determinó con ayuda del triángulo de textura del Departamento Americano de la Agricultura (USDA) (Gras, R.,1988).

\section{Estudio de los insectos dañinos}

Una cosecha se efectuó para cada árbol estudiado, con ayuda de un mariposero y de un frasco letal.

Las técnicas de cosecha eran la batida y la observación directa sobre ramas, flores y frutos del árbol. El tiempo consagrado a la tarea era por término medio de una hora por árbol la cosecha, siendo no obstante más largo sobre los individuos en floración y/ o fructificación, a causa del gran número de órganos a observar.

Después los insectos estaban pinchados, y luego determinados gracias a publicaciones anteriores sobre los principales enemigos del Prosopis (Aguilera, A., Bobadilla, D., Cortés, R., Vargas, 1986; Bobadilia, D. Cortés, R., Vargas, 1987; Vargas, H., Bobadilla, 2002 ; Nunez-Sacarias, 1993), pero también con la ayuda de profesores de Enthomologie de las Universidades de Tacna y Arica.

\section{Análisis estadistico}

Las herramientas estadisticas utilizadas se resumen en la "correlación por rango " (Spearman Rank Correlations, Statgraf), dado el número demasiado pequeño de individuos estudiados que no permitiań la utilización de la correlación simple (Correlations, Statgraph). Los resultados se calculaban con un riesgo de primera especie de $5 \%$.

Por lo que se refiere a los datos cualitativos, se trataron con ayuda de gráficos bajo Excel.

\section{RESULTADOS}

El conjunto de los resultados relativos a los caracteres morfológicos de los árboles estudiados se presenta en el Cuadro $\mathrm{N}^{\circ} 2$. Los comentarios se organizan a continuación según los grupos de variables presentados en el capitulo anterior, Material y Métodos.

Cuadro $\mathrm{N}^{\circ} \mathrm{O2}$

Caracteres morfológicos de los individuos estudiados

\begin{tabular}{|c|c|c|c|c|c|c|c|c|c|c|c|}
\hline Ind. & A.Tot & A.Tronc & Area.Copa & L.Med.Pin & Num.Par.Fol & Long.Fol & Anch.Fol & Eap.Fol & Ram.Jov & & \\
\hline$\sqrt{1}$ & $0,5 \mathrm{~m}$ & $0 \mathrm{~m}$ & $23,0 \mathrm{~m} 2$ & $5,6 \mathrm{~cm}$ & 21,8 & $0,8 \mathrm{~cm}$ & $2.0 \mathrm{~mm}$ & $2,6 \mathrm{~mm}$ & & $\sqrt[A]{A}$ & A \\
\hline 2 & $2,0 \mathrm{~m}$ & $0 \mathrm{~m}$ & $87.0 \mathrm{~m} 2$ & $6,4 \mathrm{~cm}$ & 23 & $1,1 \mathrm{~cm}$ & $2,1 \mathrm{~mm}$ & $2,8 \mathrm{~mm}$ & & $3 \longdiv { P }$ & P \\
\hline 3 & $2,2 \mathrm{~m}$ & $0 \mathrm{~m}$ & $212,5 \mathrm{~m} 2$ & $8,5 \mathrm{~cm}$ & 25,4 & $1,1 \mathrm{~cm}$ & $1.9 \mathrm{~mm}$ & $3,9 \mathrm{~mm}$ & & P & P \\
\hline 4 & $2,5 \mathrm{~m}$ & $0 \mathrm{~m}$ & $18,3 \mathrm{~m}^{2}$ & $6,7 \mathrm{~cm}$ & 21 & $1,5 \mathrm{~cm}$ & $2,5 \mathrm{~mm}$ & $3.5 \mathrm{~mm}$ & & $\sqrt[A]{A}$ & $\mathrm{P}$ \\
\hline 5 & $2,1 \mathrm{~m}$ & $0 \mathrm{~m}$ & $29.5 \mathrm{~m} 2$ & $6.0 \mathrm{~cm}$ & 19,8 & $0,8 \mathrm{~cm}$ & $2,1 \mathrm{~mm}$ & $3,0 \mathrm{~mm}$ & & $2 \mathrm{P}$ & A \\
\hline 6 & $0,7 \mathrm{~m}$ & $0 \mathrm{~m}$ & $17,4 \mathrm{~m} 2$ & $4.2 \mathrm{~cm}$ & 17 & $0,7 \mathrm{~cm}$ & $1,8 \mathrm{~mm}$ & $2,4 \mathrm{~mm}$ & & $2 \mid A$ & $\bar{A}$ \\
\hline 7 & $0,5 \mathrm{~m}$ & $0,6 \mathrm{~m}$ & $76,3 \mathrm{~m} 2$ & $4.1 \mathrm{~cm}$ & 17,6 & $0.7 \mathrm{~cm}$ & $1,7 \mathrm{~mm}$ & $3,5 \mathrm{~mm}$ & & $1 \mathrm{~A}$ & A \\
\hline 8 & $4,0 \mathrm{~m}$ & $2,8 \mathrm{~m}$ & $38,6 \mathrm{~m} 2$ & $7,7 \mathrm{~cm}$ & 19,8 & $1.1 \mathrm{~cm}$ & $1,8 \mathrm{~mm}$ & $3,6 \mathrm{~mm}$ & & $3 P$ & P \\
\hline 9 & $2,2 \mathrm{~m}$ & $1,2 \mathrm{~m}$ & $30,1 \mathrm{~m} 2$ & $5,0 \mathrm{~cm}$ & 10 & $1,1 \mathrm{~cm}$ & $2,5 \mathrm{~mm}$ & $4,0 \mathrm{~mm}$ & & $1 / \mathrm{A}$ & A \\
\hline Media & $1,9 \mathrm{~m}$ & $0,5 \mathrm{~m}$ & $59,2 \mathrm{~m}$ & $6,0 \mathrm{~cm}$ & 20,4 & $1,0 \mathrm{~cm}$ & $2,0 \mathrm{~mm}$ & $3,0 \mathrm{~mm}$ & 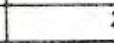 & 2 & \\
\hline
\end{tabular}




\section{Arquitectura del árbol}

Por lo que se refiere a las variables relativas a la arquitectura del Prosopis chilensis, se puede observar que el árbol es, en general, de pequeño tamaño, su altura variando de $0,7 \mathrm{a} 4 \mathrm{~m}$. La mayor parte del tiempo, no presenta tronco (a excepción de los individuos $7, .8$ y 9), sino ramificaciones que crecen directamente del suelo.

El área de copa es muy variable, dependiendo de los individuos, alcanzando $212.5 \mathrm{~m} 2$ en el individuo 3 , estas variaciones no presentan ninguna relación notable con la altura total del árbol.

Como norma general, el Prosopis chilensis presenta una forma de matorral más o menos desarrollado.

\section{Hojas}

Las pinnas tienen una longitud media de $6.0 \mathrm{~cm}$; las más pequeñas $(4.1 \mathrm{y} 4.2 \mathrm{~cm})$ proceden de los individuos 6 y 7 del valle de Sama, mientras que las más largas $(8.5 \mathrm{~cm})$ se registran en el individuo 3 en el valle de Caplina.

El número de pares de folíolos, que tiene como media 20.4 pares por pinnas, se conecta directamente a la longitud de las pinnas.

Por otra parte, por lo que se refiere al tamaño de los foliolos, los valores máximos se registraron en los individuos 4 (Long $=1.5 \mathrm{~cm}$ et $\operatorname{Larg}=2.5 \mathrm{~mm}$ ) y 9 (Long=1.1 $\mathrm{cm}$ et $\mathrm{Larg}=2.5 \mathrm{~mm}$ ); los otros individuos tienen hojas cuya longitud oscila alrededor de $1 \mathrm{~cm}$ y el ancho alrededor de $2 \mathrm{~mm}$.

Como norma general, se puede observar que los individuos del valle de Sama presentan hojas y folíolos de tamafio más pequeño con relación a los otros individuos, ver Figura 1.

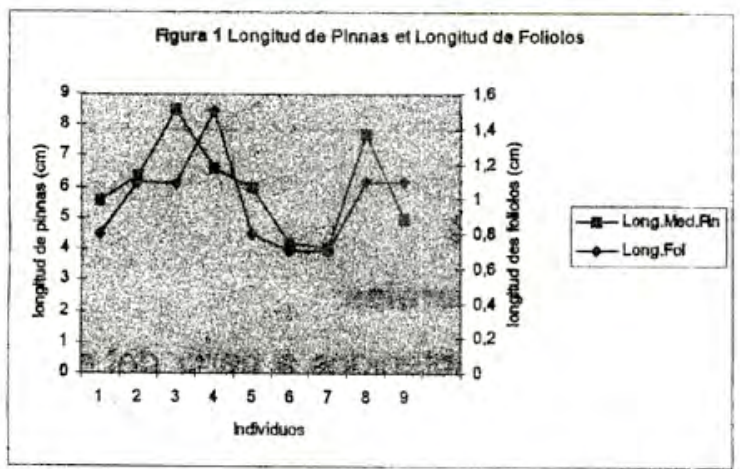

El espacio entre folíolos varía según los individuos, de 2.4 a $4.0 \mathrm{~mm}$., siempre superior a la anchura de los foliolos, pero sin conexión directa con ésta.

Por último. los árboles del valle de Caplina. a excepción del individuo 1. presentan una cantidad bastante elevada de ramos jóvenes, 10 mismo ocurre con el individuo 8. Sin embargo, los árboles no están generalmente en período de brote, presentando en su mayoría ramos del año anterior; incluso los individuos 6 y 7 del valle de Sama son casi secos.

\section{Flores y Frutos}

Las flores y frutos están la mayor parte del tiempo presentes simultáneamente, a excepción en los individuos 4 y 5 . En general, se desarrollan sobre árboles en periodo de foliación, pero no están nunca presentes en cantidad muy importante.

\section{Suelos}

Los resultados obtenidos a partir de las muestras de suelo se presentan en el Cuadro 3.

Cuadro $\mathrm{N}^{\circ} 03$

Características edáficas de los distintos lugares

\begin{tabular}{|c|c|c|c|c|}
\hline ind. & $\mathrm{Ph}$ & Cond.Eléc & Porc.Grava & Textura \\
\hline 1 & 7,6 & $9,9 \mathrm{mmos} / \mathrm{cm}$ & $43,0 \%$ & $\mathrm{FA}^{*}$ \\
\hline 2 & 7,9 & $5,3 \mathrm{mmos} / \mathrm{cm}$ & $19,0 \%$ & FA \\
\hline 3 & 8,1 & $7,1 \mathrm{mmos} / \mathrm{cm}$ & $22,2 \%$ & $F A$ \\
\hline 4 & 8 & $6,0 \mathrm{mmos} / \mathrm{cm}$ & $43,4 \%$ & $\widehat{A F^{* \infty}}$ \\
\hline 5 & 7,7 & $12,9 \mathrm{mmos} / \mathrm{cm}$ & $39,0 \%$ & $\mathrm{AF}$ \\
\hline 6 & 8 & $2,0 \mathrm{mmos} / \mathrm{cm}$ & $11,6 \%$ & $\mathrm{~A}^{* \star *+}$ \\
\hline 7 & 7,4 & $1,7 \mathrm{mmos} / \mathrm{cm}$ & $28,2 \%$ & AF \\
\hline 8 & 7,6 & $0,7 \mathrm{mmos} / \mathrm{cm}$ & $43,2 \%$ & AF \\
\hline 9 & 7,6 & $1,6 \mathrm{mmos} / \mathrm{cm}$ & $12,0 \%$ & $F A$ \\
\hline Moy & 7,8 & $5,3 \mathrm{mmos} / \mathrm{cm}$ & $30 \%$ & \\
\hline
\end{tabular}

${ }^{*} \mathrm{FA}=$ Franco Arenoso

${ }^{* *} \mathrm{AF}=$ Arena Franca

${ }^{\star \star \star *} A=$ Arena

Los suelos estudiados son todos básicos, con un $\mathrm{pH}$ variando poco entre los lugares y yendo de 7.4 a 8.1. El análisis de la conductividad eléctrica nos indica suelos salinos a extremadamente salinos (ind. 5 , Cond. Eléc. $=12.9 \mathrm{mmos} / \mathrm{cm}$ ) en el valle de Caplina. El elevado valor de conductividad eléctrica de la muestra 5 es tanto más sorprendente dado que la muestra 4 tomada a proximidad (alrededor de $7 \mathrm{~m}$ ), tiene una conductividad eléctrica claramente menos elevada. Esto puede ser explicado Dor una contaminación de suelo que viene de la carretera o la explotación agraria vecina. 
Al contrario, los suelos de los valles de Sama y Cinto se consideran como normales con valores de conductividad eléctrica que van de 0.7 a $2 \mathrm{mmos} / \mathrm{cm}$.

Los suelos son en general pedregosos, alcanzando un $43 \%$ de grava en las muestras 1,4 y 8 . Por fin, las texturas de suelo encontradas, Franco Arenoso, Arena Franca y Arena, son bastante cercanas, teniendo como carácter común un porcentaje elevado en arena.

Relación entre variabilidad morfológica y factores ecológicos.

El test de correlación por rango se utilizó para poner de relieve correlaciones entre variables cuantitativas de los siguientes grupos:

Arquitectura del árbol/Suelo

Arquitectura del árbol/Clima

Hoja/Suelo

Hoja/Clima

Una correlación significativa solo existe entre un número muy pequeño de variables que figuran a continuación.

Arquitectura/Suelo: No hay correlación entre las variables

Arquitectura/Clima: Altura del Tronco correlacionada con Temperatura media, $\mathrm{C}=0,8214$ y $\mathrm{p}=0,0202$

Altura total correlacionada con Precipitación media, $C=-0,7967$ y $p=0,0242$ (ver Figura 2)

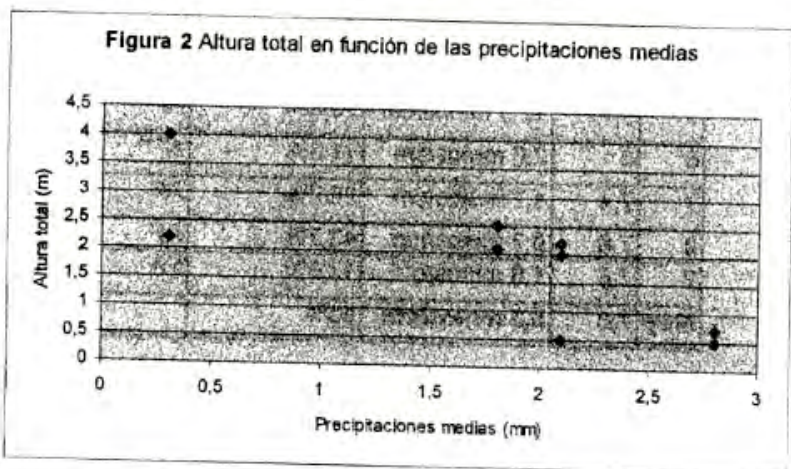

Hojas/Clima: Longitud de foliolos correlacionada con Precipitación media, $\mathrm{C}=-0,7357$ y 0,0374

\section{Insectos dañinos}

Los insectos recolectados pertenecen a los órdenes Coleóptera, Hymenóptera y Lepidóptera.

Según la Figura 3 , se puede observar que las familias de insectos más frecuentes son los Psilideae y los Tortricideae, presentes en todos los árboles estudiados. Las otras familias representadas se encuentran en menor cantidad.

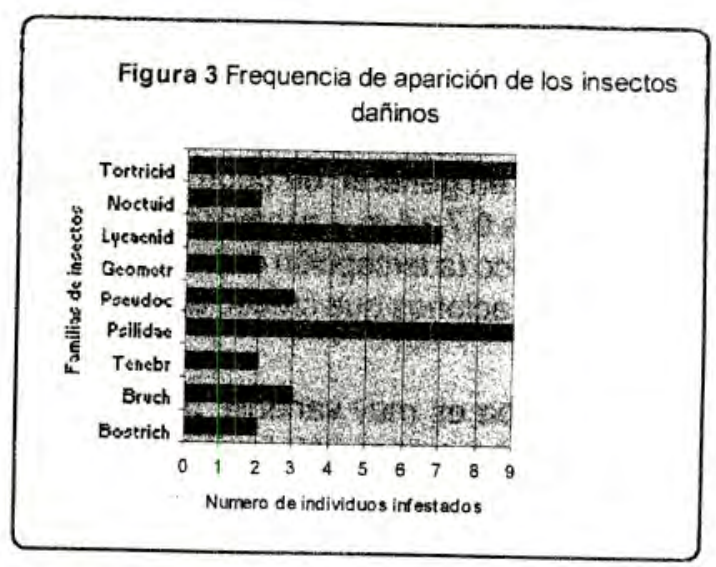

Por otra parte, el número de familias de insectos recolectados es especialmente elevado en los individuos 2,3 y 5 (6 a 9 familias); esto puede ponerse en relación directa con la presencia de flores y frutos sobre estos árboles, ver Figura 4. En efecto, algunas familias como los Bostrichideae, Bruchideae y los Tenebrionideae se alimentan esencialmente de frutos, mientras que Lycaenideae y Geometrideae tienen una preferencia por las flores. Al contrario, los Psilideae, como chupador de savia y los Tortricideae, cuyas larvas de algunas especies se alimentan con hojas, están presentes en todos los individuos.

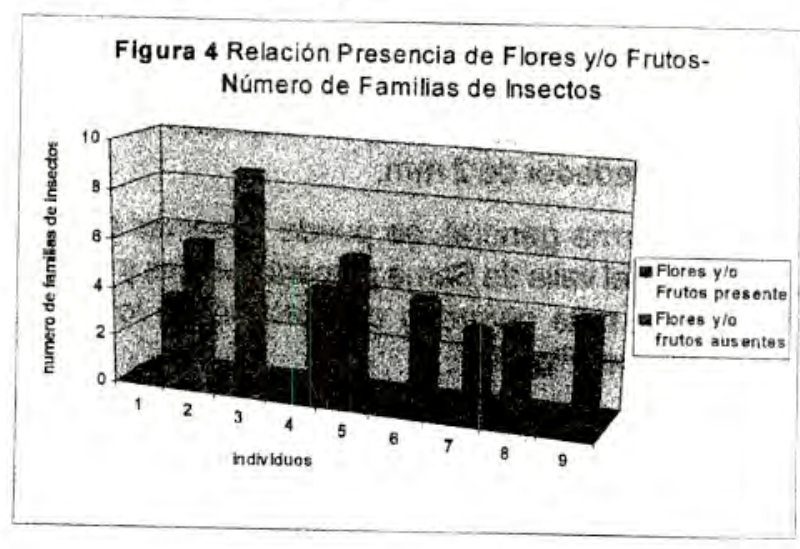

\section{DISCUSIONES}

\section{Caracteres generales}

Los resultados obtenidos a partir del estudio morfológico de Prosopis chilensis están como norma general comparables a las características del árbol contabilizadas en publicaciones anteriores. Sin embargo, la mayor parte del tiempo, los individuos estudiados presentan algunos caracteres de pequeño tamaño con relación a la norma, es decir, cerca de los valores inferiores contabilizados. Tomemos como ejemplo el carácter tamaño del árbol, éste se 
calibra de $0,5 \mathrm{~cm}$ a $4,0 \mathrm{~cm}$ en la experimentación, mientras que el margen presentado para la especie es de 3,0 a 10,0 m (Burkart, A., 1976; Ffolliot, P.F. , Thames, J.L., 1983 ; Trobock, S., 1987). Lo mismo ocurre con la longitud de las pinnas que miden normalmente entre 8 y $24 \mathrm{~cm}$ (Ffolliot, P.F., Thames,J.L., 1983), pero que en la experimentación no sobrepasan el $8,5 \mathrm{~cm}$, presentando valores claramente inferiores a los datos teóricos. Se puede observar el mismo fenómeno para la longitud, la anchura y el espacio entre los foliolos que presentan valores especialmente pequeños.

Por otra parte, las hojas de los individuos estudiados sólo estaban constituidas por un único par de pinnas, mientras que las distintas publicaciones mencionan por especie 1 a 3 pares de pinnas. Estas diferencias con relación a las normas pueden ser justificadas por el hecho de que éstas se contabilizaron sobre individuos que crecian en condiciones no perturbadas (Ffolliot,P.F., Thames, J.L., 1983). Aunque la región de Tacna, situada en el desierto de Atacama presente condiciones ecológicas especialmente extremas (Chávez 2002). El bajo tamaño de los caracteres observados podría pues explicarse por una adaptación morfológica del Prosopis chilensis al clima desértico de la región de Tacna. Sin embargo, sería interesante verificar por un análisis genético si los árboles estudiados no pertenecen a una nueva variedad.

Se puso de relieve también una variabilidad morfológica entre los individuos estudiados. Esta variabilidad podría vincularse con una adaptación a las condiciones ecotógicas propias de cada lugar, hipótesis que vamos a analizar en los acápites siguientes, estudiando inicialmente los resultados de los tests de correlación, para retrasarse a continuación sobre los caracteres cualitativos del árbol.

\section{Arquitectura del árbol y factores ecológicos}

La arquitectura del árbol y su relación con el suelo.

La aproximación de las variables relativas a la arquitectura del árbol y los factores edáficos, gracias al test de correlación por rango, no puso de relieve ninguna correlación. Este resultado indica que los factores edáficos no determinarian directamente la arquitectura del árbol. Por lo que se refiere a las variables ph y textura, la ausencia de correlación con las variables morfológicas puede ser explicada por las pequeñas diferencias encontradas en el $\mathrm{pH}$ y la textura entre los distintos suelos. Además, los valores $\mathrm{pH}$ y textura medidos corresponden a las caracteristicas de suelo recomendadas para el cultivo del Prosopis (Diaz, C., 1995).

Por lo que se refiere a las variables conductividad eléctrica y Pourc. de Grava, dado que la variación entre las muestras es más importante, la ausencia de correlación puede ser justificada por una buena resistencia de Prosopis chilensis a la salinidad del suelo y por una insensibilidad al carácter pedregoso de los suelos. En efecto, esta especie se conoce por su capacidad para desarrollarse sobre suelos extremadamente salinos por una parte, y también pedregosos, hasta rocosos (Díaz, C., 1995). Sin embargo, el individuo 5 , situado cerca del individuo 4 (aproximadamente $7 \mathrm{~m}$ ), pero creciendo sobre un suelo claramente más salino (Conductividad eléctrica $=12.9 \mathrm{mmos} / \mathrm{cm}$, las otras características edáficas siendo casi idénticas) presenta un estado sanitario claramente más degradado; es decir, pocos ramos jóvenes, no fructificación, y sufriendo un ataque de insectos dañinos especialmente fuerte. Se podría pues suponer que una elevada salinidad aumentaría la sensibilidad de los árboles a los ataques de insectos enemigos(Díaz, C., 1995). En efecto, se probó que dosis extremas en nitrógeno favorecen el ataque de insectos y ácaros. Ahora bien, la úrea aumenta la salinidad de los suelos.

Según los tests de correlación por rango, existe una correlación negativa entre las variables altura total del árbol y precipitación media. Al dar este resultado por válido, se puede intentar explicar la influencia negativa de las precipitaciones sobre el crecimiento vertical del árbol, por un desarrollo privilegiado de su sistema de raíz horizontal. En efecto, Prosopis chilensis, como especie freatofito facultativo, desarrolla un primer sistema de raices formado por una raiz pivotante que puede alcanzar hasta $58 \mathrm{~m}$ y favoreciendo una alimentación hídrica en la napa freática. Mientras que un segundo sistema de raíces, formado por raices horizontales, permite al árbol recuperar el agua de las lluvias (Cony, M., 1998), (ver Figura 5 en anexo). Así, al considerar que el desarrollo de raiz tiene una influencia directa sobre el 
crecimiento aéreo del árbol, se podría explicar el crecimiento horizontal preferencial de los árboles del valle de Sama por la ausencia supuesta de napa freática. En efecto, estos árboles que crecen sobre una parte alta del cerro, lo que supone una capa freática ausente o muy profunda, habrian desarrollado preferencialmente un sistema de raíces horizontales con el fin de optimizar la captación del agua de las lluvias. Al contrario, los individuos 8 y 9 del valle de Cinto que crecen en el medio de la avenida aluvial, lugar donde la capa freática se supone de poca profundidad, habrian desarrollado preferencialmente su raiz pivotante para abastecerse en la capa freática y son, pues, especialmente altos con relación a los otros individuos. Por otra parte, según los resultados, la altura del tronco se correlaciona con la temperatura media; sin embargo, esta correlación es poco fiable dado el gran número de árboles sin tronco encontrado (un número importante de ceros puede falsificar el análisis estadístico). La presencia de tronco en algunos individuos (ind. 7,8 y 9) podría ser explicada por una intervención humana (tamaño). En efecto, según un estudio efectuado sobre una plantación de Prosopis efectuada en el departamento de Tacna, la tendencia natural de Prosopis chilensis sería más bien desarrollar varias ramificaciones a partir del suelo ( 3 a 6 ramificaciones principales contabilizadas sobre árboles de 3 años y medio de edad, Cassana Torres, F.A., 1992).

Por otra parte, el área de copa puede también ser limitado por una competencia en el espacio con los vegetales circundantes, y también por podas del árbol por lo que se refiere a los individuos situados cerca de chacras (ind. $1, .2,3, .4$ y 5$)$.

\section{Características de las hojas y factores ecológicos}

Los tests de correlación no pusieron de relieve ninguna relación entre las características de las hojas y los factores edáficos.

Pero, según los resultados, existiria una correlación negativa entre longitud de los folíolos y precipitaciones medias. Este resultado sigue siendo poco pausible, ya que se opone a las teorías anteriores que preven una reducción de la superficie foliar con el fin de que absorban menos energía para evitar el déficit hidrico (Cony, M., 1998).

Como norma general, el conjunto de los resultados obtenidos a partir de estas correlaciones sigue siendo poco fiable y no permite llegar a conclusiones, dado el escaso número de individuos estudiados, a causa de dificultades temporales y espaciales (elevada distancia entre los distintos valles).

\section{Estudio de los caracteres cualitativos}

Inicialmente, se pueden observar diferencias obvias en la cantidad de ramos jóvenes entre los individuos (ver Cuadro 2). Por otra parte, se observa esta variabilidad en la fase de foliación en individuos muy cercanos, como en Calana donde el individuo 1 está en fase 2, mientras que los individuos 2 y 3 están en fase 3 . Este fenómeno podría explicarse como un mecanismo de adaptación morfológico para tolerar el déficit hídrico en zonas especialmente secas donde la napa freática es ausente o muy profunda. En efecto, en condiciónes no perturbadas el Prosopis presenta dos flujos de foliación, un primero al principio de la primavera, y un segundo, menos importante, más cerca del verano. Pero, en los medios más áridos, se observó que el segundo flujo solo tenia lugar en un número de árboles muy escaso (Cony, M., 1998).

Por otra parte, es posible poner en relación esta variabilidad en la foliación con los fenómenos de floración y fructificación. En efecto, los resultados nos indican que solamente los individuos en período de foliación producen flores y/o frutos en cantidad notable (ind. 5 excluido, presentan un número muy escaso de flores). Considerando el período de estudio como un período de floración y fructificación secundaria (el período principal teniendo lugar en verano, Díaz, C., 1995), solamente los árboles en actividad fisiológica producen flores y/o frutos.

Por otra parte, como la famosa Figura 5 , los individuos en foliación, floración y/o fructificación sufren un ataque más importante de insectos devastadores, ya que responden a una gama más amplia de regímenes alimentarios.

Para concluir, se puede separar el conjunto de los individuos estudiados en dos grupos, un primero agrupando a los individuos $2,3,4$ y 8 , con 
árboles que presentan un buen desarrollo y una actividad fisiológica que dura las temporadas otoñal e invernal; un segundo grupo que reúne los individuos $1,6,7$ y 9 , que se desarrollan claramente menos y están en descanso vegetativo durante la mala temporada. Se clasifica al individuo 5 a un lado, ya que que presenta un buen desarrollo vegetativo, pero una escasa actividad fisiológica, seguramente vinculada al fuerte ataque de insectos que sufre. Esta separación en 2 grupos parece sobre todo determinada por la disponibilidad de agua del suelo.

Desgraciadamente, la experimentación llevada no permite poner de relieve relaciones válidas entre estas diferencias morfológicas observadas y los factores ecológicos estudiados, a causa de un número demasiado escaso de árboles estudiados. Sin embargo, sería interesante reanudar esta experimentación sobre un mayor número de individuos y efectuando un análisis de los caracteres edáficos más profundo (corte de terreno sobre $1 \mathrm{~m}$ de profundidad, análisis de la humedad del suelo y la profundidad de la napa freática).

Por otra parte, dadas las diferencias encontradas entre las características de los árboles estudiados y las normas indicadas en las publicaciones, un análisis genético sería necesario con el fin de verificar si los individuos estudiados no pertenecen a una nueva variedad.

\section{REFERENCIAS BIBLIIOGRÁFICAS}

1. Aguilera, A., Bobadilla, D., Cortés, R., Vargas, H., 1986. Proyecto: Estudio de insectos que atacan al tamarugo (Prosopis tamarugo) y algarrobo (Prosopis chilensis). Segundo informe sobre el desarrollo del proyecto presentado por la Universidad de Tarapacá (Chile), et al. Instituto de Agronomia (IDEA). 6-37p

2. Bobadilla, D.; Cortés, R.; Vargas, H. 1987. Proyecto: Estudio de insectos que atacan tamarugo (Prosopis tamarugo Phil) y algarrobo [Prosopis chilensis (Mol.) Stuntz] Informe final sobre el desarollo del proyecto presentado par la Universidad de Tarapacá (Chile), et al. Instituto de Agronomia (IDEA).

3. Burkart, A., 1976. A monograph of the genus Prosopis (Mimosoideae). Journal of Arnold Arboretum, 57(3): 219-249.

4. Cassana Torres, F.A., 1992. Ensayo de procedencias de Prosopis pallida (H. et B. ex Willd) H.B.K., Prosopis tamarugo Phil. y Prosopis chilensis (Mol.) Stuntz, en Tacna. Thèse de doctorat, Université de Huancayo.

5. Contreras, B., 1987. Morphological diversity in Prosopis chilensis populations aand evaluation of its growth in plantalon at region IV. In: Estudio de insectos que atacan tamarugo y algarrobo. Bobadilla, D., Cortés, R., Vargas, H., 1987. 311-331 p.

6. Cony, M., 1998. Importancia de los estudios fisiológicos, ecofisiológicos y genéticos sobre especies del género Prosopis para la recuperación de ecosistemas áridos degradados de América Latina. En : Proyecto Algarrobo. INRENA. Memorias del Seminario Internacional. Lima, Perú. Cap IV- 255,262, $264 \mathrm{p}$.

7. Chávez, R, 2002 Mejoramiento genético de plantas tuberíferas para zonas árido - salinas, Edit. Art. Graphics, $330 \mathrm{pd}$.

8. Diaz, C., 1995. Los algarrobos. Consejo Nacional de Ciencia y Tecnología. Lima.64, 73-74, 83p.

9. Ffolliot, P.F., Thames, J.L., 1983. Manual sobre la taxonomía de Prosopis en México, Perú y Chile.

10. Gras, R., 1988. Physique du sol pour l'aménagement. $34 \mathrm{p}$.

11. Morizaki Taura, A., 1998. Politica de manejo de lps bosques secos. En : Proyecto Aigarrobo. INRENA. Memorias del Seminario Internacional. Lima, Perú. Capl-4,5p.

12. Morris Ayca, M., Panty Neyra, O., 1999. Espacio y conciencia geográfica en Tacna. 109,110 p.

13. Nunez-Sacarias, E., 1993. "Insectos del algarrobo ( Prosopis spp) en el Perú: costa norte (Piura) y costa central (Ica)". Rev. per. Ent. 36: 69-83 p

14. Trobok, S., 1987. Fruit and seed morphology of Chilean Prosopis (Fabaceae-Mimosoideae). En: Estudio de insectos que atacan tamarugo y algarrobo. Bobadilla, D., Cortés, R., Vargas, H., 1987. 227-262 p.

15. Vargas, H., Bobadilla, D., 2002. Insectos asociados al bosque de tamarugo. En: Agentes de daño en el bosque nativo. Editores: A. Baldini \& L. Pancel. p. $283-318$. Editorial Universitaria. Santiago de Chile.

16. Zegarra Z. Rosario 1994. "La vegetación desértica perenne de Tacna: Estudio biosistemático y sus recursos naturales". Revista Nueva Imagen. Universidad Nacional Jorge Basadre Grohamnn de Tacna - Perú. Págs. 53-66

17. Zegarra Z. Rosario 2003. "Vegetación desértica del valle de cinto". Revista Ciencia y Desarrollo $N^{\circ} 07$, Universidad Nacional Jorge Basadre Grohmann de Tacna - Perú. 